\title{
Electromagnetic Wave Functions of CMB and Schwarzschild Space-Time
}

\author{
Sangwha-Yi
}

Department of Math, Taejon University 300-716

\begin{abstract}
*Corresponding Author: Sangwha-Yi, Department of Math, Taejon University 300-716
\end{abstract}
Abstract: In the general relativity theory, we find electro-magnetic wave functions of Cosmic Microwave Background and Schwarzschild space-time. Specially, this article is that electromagnetic wave equations are treated by gauge fixing equations in Robertson-Walker space-time and Schwarzschild space-time.

Keywords: General relativity theory, Electro-magnetic wave equations; Electromagnetic wave functions; Cosmic Microwave Background; Schwarzschild space-time

PACS Number: 04,04.90. $+e, 03.30,41.20$

\section{INTRODUCTION}

In the general relativity theory, our article's aim is that we find electro-magnetic wave equations and functions by gauge fixing equations in Robertson-Walker space-time and Schwarzschild space-time.

At first, Electro-magnetic field equations are in general relativity theory

$$
\begin{gathered}
F_{i v}^{\mu v}=\frac{4 \pi}{c} j^{\mu} \\
F_{\mu v ; \lambda}+F_{v \lambda ; \mu}+F_{\lambda \mu i v}=0
\end{gathered}
$$

The electro-magnetic field is

$$
F_{\mu \nu}=A_{v ; \mu}-A_{\mu i v}=\frac{\partial A_{v}}{\partial x^{\mu}}-\frac{\partial A_{\mu}}{\partial x^{v}}
$$

The gauge fixing equation in general relativity theory

$$
\begin{aligned}
& A^{\mu}{ }_{; \mu}=\frac{\partial A^{\mu}}{\partial x^{\mu}}+\Gamma_{\mu \rho}^{\mu} A^{\rho} \\
\rightarrow & \partial_{\mu}\left(A^{\mu}+g^{\mu v} \partial_{\nu} \Lambda\right)+\Gamma_{\mu \rho}^{\mu}\left(A^{\rho}+\partial^{\rho} \Lambda\right) \\
= & \partial_{\mu}\left(A^{\mu}+g^{\mu v} \partial_{v} \Lambda\right)+\Gamma_{\mu \rho}^{\mu}\left(A^{\rho}+g^{\rho \rho} \partial_{\rho} \Lambda\right)
\end{aligned}
$$

\section{ELECTRO-MAGNETIC WAVE EQUATION IN ROBERTSON-WALKER SPACE-TIME}

Because the gauge fixing equation is the electro-magnetic wave equation, the electro-magnetic wave equation is in Robertson-Walker space-time. 
The Robertson-Walker solution is

$d \tau^{2}=d t^{2}-\frac{1}{c^{2}} \Omega^{2}(t)\left[\frac{d r^{2}}{1-k r^{2}}+r^{2} d \Omega^{2}\right]$

In this time, 2-dimensional solution is

$d \Omega=0$

$d \tau^{2}=d t^{2}-\frac{1}{c^{2}} \Omega^{2}(t) \frac{d r^{2}}{1-k r^{2}}$

The gauge fixing equation is in 2-dimensional solution

$$
\begin{aligned}
& \partial_{\mu}\left(A^{\mu}+g^{\mu v} \partial_{v} \Lambda\right)+\Gamma^{\mu}{ }_{\mu \rho}\left(A^{\rho}+g^{\rho \rho} \partial_{\rho} \Lambda\right) \\
& =\partial_{\mu} A^{\mu}+\Gamma^{1}{ }_{10} A^{0}+\Gamma^{1}{ }_{11} A^{1}+\partial_{\mu} g^{\mu v} \partial_{v} \Lambda+g^{\mu v} \partial_{\mu} \partial_{v} \Lambda+\Gamma^{1}{ }_{10} g^{00} \frac{1}{c} \frac{\partial \Lambda}{\partial t}+\Gamma^{1}{ }_{11} g^{11} \frac{\partial \Lambda}{\partial r}
\end{aligned}
$$

Hence, we can find electro-magnetic wave equation in 2-dimentional Robertson-Walker space-time.

$$
\begin{aligned}
& \partial_{\mu} g^{\mu v} \partial_{v}(\sin \Phi)+g^{\mu v} \partial_{\mu} \partial_{v}(\sin \Phi)+\Gamma_{10}^{1} g^{00} \frac{1}{c} \frac{\partial}{\partial t}(\sin \Phi)+\Gamma_{11}^{1} g^{11} \frac{\partial}{\partial r}(\sin \Phi) \\
& =\left[\frac{-2 k r}{\Omega^{2}(t)} \frac{\partial}{\partial r}-\frac{1}{c^{2}} \frac{\partial^{2}}{\partial t^{2}}+\frac{1-k r^{2}}{\Omega^{2}(t)} \frac{\partial^{2}}{\partial r^{2}}-\frac{\dot{\Omega}}{c \Omega} \frac{1}{c} \frac{\partial}{\partial t}+\frac{k r}{\Omega^{2}(t)} \frac{\partial}{\partial r}\right] \sin \Phi=0 \\
& \Gamma_{10}^{1}=\frac{\dot{\Omega}}{c \Omega} \quad, \quad \Gamma^{1}{ }_{11}=\frac{k r}{1-k r^{2}}
\end{aligned}
$$

In this time, we can think the shape of electro-magnetic wave function from 2-dimetional RobertsonWalker space-time. In this case, light is

$$
\begin{aligned}
& d \tau^{2}=d t^{2}-\frac{1}{c^{2}} \Omega^{2}(t) \frac{d r^{2}}{1-k r^{2}}=0 \\
& \int \frac{d t}{\Omega(t)}=\frac{1}{c} \int \frac{d r}{\sqrt{1-k r^{2}}}
\end{aligned}
$$

Hence, electro-magnetic wave function is in 2-dimetional Robertson-Walker space-time-

$\vec{E}=\vec{E}_{0} \sin \Phi, \vec{B}=\vec{B}_{0} \sin \Phi$

$\Phi=\omega_{0}\left[\int \frac{d t}{\Omega(t)}-\frac{1}{c} \int \frac{d r}{\sqrt{1-k r^{2}}}\right]$

i) $k=1, \Phi=\omega_{0}\left[\int \frac{d t}{\Omega(t)}-\frac{1}{c} \sin ^{-1} r\right]$

ii) $k=0, \Phi=\omega_{0}\left[\int \frac{d t}{\Omega(t)}-\frac{1}{c} r\right]$

iii) $k=-1, \Phi=\omega_{0}\left[\int \frac{d t}{\Omega(t)}-\frac{1}{c} \sinh ^{-1} r\right]$

The electro-magnetic wave equation- $\mathrm{Eq}(8)$ is satisfied by the electro-magnetic wave function- $\mathrm{Eq}(10)$.

\section{ELECTRO-MAGNETIC WAVE EQUATION IN SCHWARZSCHILD SPACE-TIME}

Because the gauge fixing equation is the electro-magnetic wave equation, the electro-magnetic wave equation is in Schwarzschild space-time. 
The Schwarzschild solution is

$$
d \tau^{2}=\left(1-\frac{2 G M}{r c^{2}}\right) d t^{2}-\frac{1}{c^{2}}\left[\frac{d r^{2}}{1-\frac{2 G M}{r c^{2}}}+r^{2} d \Omega^{2}\right]
$$

In this time, 2-dimensional solution is

$$
\begin{aligned}
& d \Omega=0 \\
& d \tau^{2}=\left(1-\frac{2 G M}{r c^{2}}\right) d t^{2}-\frac{1}{c^{2}} \frac{d r^{2}}{1-\frac{2 G M}{r c^{2}}}
\end{aligned}
$$

The gauge fixing equation is in 2-dimensional solution

$$
\begin{aligned}
& \partial_{\mu}\left(A^{\mu}+g^{\mu v} \partial_{v} \Lambda\right)+\Gamma^{\mu}{ }_{\mu \rho}\left(A^{\rho}+g^{\rho \rho} \partial_{\rho} \Lambda\right) \\
& =\partial_{\mu} A^{\mu}+\Gamma^{0}{ }_{01} A^{1}+\Gamma^{1}{ }_{11} A^{1}+\partial_{\mu} g^{\mu v} \partial_{v} \Lambda+g^{\mu v} \partial_{\mu} \partial_{v} \Lambda+\Gamma^{0}{ }_{01} g^{11} \frac{\partial \Lambda}{\partial r}+\Gamma^{1}{ }_{11} g^{11} \frac{\partial \Lambda}{\partial r} \\
& =\partial_{\mu} A^{\mu}+\partial_{\mu} g^{\mu v} \partial_{v} \Lambda+g^{\mu v} \partial_{\mu} \partial_{v} \Lambda \\
& \Gamma_{01}^{0}=\frac{G M}{r^{2} c^{2}} \frac{1}{1-\frac{2 G M}{r c^{2}}} \quad, \quad \Gamma^{1}{ }_{11}=-\frac{G M}{r^{2} c^{2}} \frac{1}{1-\frac{2 G M}{r c^{2}}}
\end{aligned}
$$

Hence, we can find electro-magnetic wave equation in 2-dimentional Schwarzschild space-time.

$$
\begin{aligned}
& \partial_{\mu} g^{\mu v} \partial_{\nu}(\sin \Phi)+g^{\mu v} \partial_{\mu} \partial_{\nu}(\sin \Phi) \\
& =\left[\frac{2 G M}{r^{2} c^{2}} \frac{\partial}{\partial r}-\frac{1}{1-\frac{2 G M}{r c^{2}}} \frac{1}{c^{2}} \frac{\partial^{2}}{\partial t^{2}}+\left(1-\frac{2 G M}{r c^{2}}\right) \frac{\partial^{2}}{\partial r^{2}}\right] \sin \Phi=0
\end{aligned}
$$

In this time, we can think the shape of electro-magnetic wave function from 2-dimetional Schwarzschild space-time. In this case, light is

$$
\begin{aligned}
& d \tau^{2}=\left(1-\frac{2 G M}{r c^{2}}\right) d t^{2}-\frac{1}{c^{2}} \frac{d r^{2}}{1-\frac{2 G M}{r c^{2}}}=0 \\
& t=\frac{1}{c} \int \frac{d r}{1-\frac{2 G M}{r c^{2}}}=\frac{r}{c}+\frac{2 G M}{c^{3}}|n| r-\frac{2 G M}{c^{2}} \mid
\end{aligned}
$$

Hence, electro-magnetic wave function is in 2-dimetional Schwarzschild space-time-

$$
\begin{aligned}
& \vec{E}=\vec{E}_{0} \sin \Phi, \vec{B}=\vec{B}_{0} \sin \Phi \\
& \Phi=\omega_{0}\left[t-\frac{r}{c}-\frac{2 G M}{c^{3}}|n| r-\frac{2 G M}{c^{2}}\right]
\end{aligned}
$$

The electro-magnetic wave equation- $\mathrm{Eq}(14)$ is satisfied by the electro-magnetic wave function- $\mathrm{Eq}(16)$. 


\section{CONCLUSION}

We find the electro-magnetic wave (CMB) equation and function in Robertson-Walker space-time.

We find the electro-magnetic wave equation and function in Schwarzschild space-time.

\section{REFERENCES}

[1] S.Yi, "Electromagnetic Field Equation and Lorentz Gauge in Rindler space-time", The African review of physics,11,33(2016)-INSPIRE-HEP

[2] S.Yi, "Electromagnetic Wave Function and Equation, Lorentz Force in Rindler space-time", International Journal of Advanced Research in Physical Science,5,9(2018)

[3] S. Weinberg, Gravitation and Cosmology(John wiley \& Sons,Inc, 1972)

[4] W. Rindler, Am.J.Phys.34.1174(1966)

[5] P. Bergman, Introduction to the Theory of Relativity (Dover Pub. Co.,Inc., New York,1976), Chapter V

[6] C. Misner, K, Thorne and J. Wheeler, Gravitation(W.H.Freedman \& Co.,1973)

[7] S. Hawking and G. Ellis, The Large Scale Structure of Space-Time(Cam-bridge University Press, 1973)

[8] R. Adler, M. Bazin and M. Schiffer, Introduction to General Relativity(McGraw-Hill,Inc.,1965)

[9] A. Miller, Albert Einstein's Special Theory of Relativity(Addison-Wesley Publishing Co., Inc., 1981)

[10] W. Rindler, Special Relativity(2nd ed., Oliver and Boyd, Edinburg,1966)

[11] Massimo Pauri, Michele Vallisner, "Marzke-Wheeler coordinates for accelerated observers in special relativity":Arxiv:gr-qc/0006095(2000)

[12] A. Einstein, "Zur Elektrodynamik bewegter K"orper”, Annalen der Physik. 17:891(1905)

Citation: Sangwha-Yi. "Electromagnetic Wave Functions of CMB and Schwarzschild Space-Time", International Journal of Advanced Research in Physical Science (IJARPS), vol. 6, no. 3, pp. 1-4, 2019.

Copyright: (C) 2019 Authors, This is an open-access article distributed under the terms of the Creative Commons Attribution License, which permits unrestricted use, distribution, and reproduction in any medium, provided the original author and source are credited. 